\title{
Measurements of non-equilibrium and equilibrium temperature behind a strong shock wave in simulated martian atmosphere
}

\author{
Xin Lin · Xi-Long Yu · Fei Li · Shao-Hua Zhang · Jian-Guo Xin · Xin-Yu Zhang
}

Received: 28 December 2011 / Revised: 9 March 2012 / Accepted: 9 March 2012

(C)The Chinese Society of Theoretical and Applied Mechanics and Springer-Verlag Berlin Heidelberg 2012

\begin{abstract}
Non-equilibrium radiation measurements behind strong shock wave for simulated Martian atmosphere are presented in this paper. The shock wave is established in a hydrogen oxygen combustion driven shock tube. Timeresolved spectra of the $\Delta v=0$ sequence of the $B^{2} \Sigma^{+} \rightarrow X^{2} \Sigma^{+}$ electronic transition of $\mathrm{CN}$ have been observed through optical emission spectroscopy (OES). A new method, which is based on fitting high resolution spectrum for rotational and vibrational temperatures measurement, is proposed to diagnose temperature distribution behind the shock wave. It is estimated that the current scheme has the maximum deviation less than $8 \%(1 \sigma)$ for vibrational temperature measurement through detailed analysis of the influence of the uncertainties of spectroscopic constants and spectral resolution. Radiation structure of the shock layer, including induction, relaxation and equilibrium process, and corresponding rotational and vibrational temperatures are obtained through time gating OES diagnostics with sub-microsecond temporal resolution. The present extensive results will strongly benefit the reaction rate estimation and computational fluid dynamics (CFD) code validation in high enthalpy Mars reentry chemistry.
\end{abstract}

Keywords Radiation measurements - Shock wave $\cdot$ Mars re-entry $\cdot$ Rotational temperature $\cdot$ Vibrational temperature

\section{Introduction}

When a spacecraft enters the Mars atmosphere, the heat shield of the vehicle will be expected to receive a strong

X. Lin · J.-G. Xin

School of Optoelectronics, Beijing Institute of Technology, 100081 Beijing, China

X.-L. Yu $\left(\bigotimes_{1}\right) \cdot$ F. Li $\cdot$ S.-H. Zhang $\left(\bigotimes_{2}\right) \cdot$ X.-Y. Zhang

State Key Laboratory of High Temperature Gas Dynamics, Institute of Mechanics, Chinese Academy of Sciences, 100190 Beijing, China

e-mail $1_{1}$ :xlyu@imech.ac.cn

e-mail 2 : shzh@imech.ac.cn radiative flux produced in the bow shock layer $[1,2]$. It has also been predicted that, below an entry speed of about $7 \mathrm{~km} / \mathrm{s}$, nearly all of the radiative heating is due to the $\mathrm{CN}$ violet $\left(B^{2} \Sigma^{+} \rightarrow X^{2} \Sigma^{+}\right)$and red $\left(A^{2} \Pi \rightarrow X^{2} \Sigma^{+}\right)$bands [3]. An accurate estimate of this radiative heat flux is therefore necessary for a proper design of the thermal protection system (TPS) [4-8]. Moreover, the radiation emission is strongly dependent on the non-equilibrium conditions present behind the shock wave, which requires implementation of thermochemical models for accurate prediction of the emitted radiation. Nevertheless, the present estimates of the radiative heating generated by $\mathrm{CN}$ radicals from such models vary greatly due to lack of critical experimental data. Among available data, the rotational and vibrational temperatures behind the shock wave are one of the most important thermodynamic quantities for shocked-induced chemistry, especially the rotational temperature, which is a key parameter in driving chemical reactions because it controls the collision probability of the gaseous particles in a chemically reacting hot gas. Therefore, an accurate measurement of the distribution of rotational and vibrational temperatures along the shock profile is very useful for the heating load estimates related to Mars entry, understanding non-equilibrium radiating phenomena, and CFD code validation.

Experiments and simulations investigations have been developed to predict the non-equilibrium and equilibrium temperatures behind a strong shock wave by a number of institutions, including NASA Ames Research Center in California $[9,10]$, Université de Provence in Marseilles, France $[11,12]$, and University of Queensland in Australia [13]. NASA Ames Research Center carried out a lot of experiments to measure the radiation behind a shock under different atmospheric conditions. Tests for entry into the atmospheres of Earth, Mars, and Titan were performed in the electric arc shock tube known as EAST. Temperatures profile in the postshock was then obtained by comparing thermochemical models with calibrated spectrograph measurements. University of Queensland also conducted calibrated 
intensity measurements in a wide range of conditions of parameters like velocity, pressure, and gas composition for Titan entry at the X2 high-enthalpy shock-tube facility, which were compared to similar measurements from the EAST facility. Experiments were also performed on Université de Provence's TCM2 facility to evaluate the time-resolved postshock intensity profiles and to measure postshock rotational and vibrational temperatures using emission spectroscopy. Time resolved emission of $\mathrm{CN}$ and $\mathrm{C}_{2}$ molecules were studied behind a strong shock on the $\mathrm{CN}$ violet, $\mathrm{C}_{2}$ Swan, and $\mathrm{CN}$ red radiative systems.

In our study, we focus on the narrowband range (387.6$388.6 \mathrm{~nm}$ ) of $\mathrm{CN}$ violet system rather than the total integrated radiative intensity over a large range of wavelength or low-resolution spectra of a wide spectral range. With very short intensified charge-coupled device (ICCD) gating time, the experimental spectra have high temporal and spatial resolutions. Temperature information can be directly obtained through a precise analysis of high-resolution experimental spectrum, and moreover, in this way we can also avoid the error caused by absolute calibration uncertainty. In addition, through the analysis of a "frozen" re-entry flowfield at a given gating time by changing the gate pulse delay, the evolution of vibrational and rotational temperatures along the shock profile in simulated Martian atmosphere can be obtained. A detailed discussion on the evaluation of the fitting method is also given.

\section{Experimental setup}

The experiments have been conducted in a hydrogen oxygen combustion shock tube facility, an overview of which is shown in Fig. 1 with essential dimensional details and emission spectroscopy measurement setup. The shock tube is composed of a driver section, a diaphragm and a driven section. The initial pressures of oxygen and hydrogen in the driver section are $0.06 \mathrm{MPa}$ and $0.59 \mathrm{MPa}$, respectively. Experiments have been carried out in a mixture of $70 \% \mathrm{CO}_{2}$ and $30 \% \mathrm{~N}_{2}$, despite the difference from the actual composition of the Martian atmosphere, such a ratio can provide enough signal for $\mathrm{CN}$ violet emission. The test gas fills the test section with $200 \mathrm{~Pa}$, and a $2 \mathrm{~mm}$ thickness aluminum diaphragm with a surface cross notch $0.9 \mathrm{~mm}$ in depth is fixed between the driver and driven sections. The contamination of the test mixture in the test section by outside air can be neglected because of its weak leakage lower than $0.1 \mathrm{~Pa} / \mathrm{min}$. In addition, before each new filling, the shock tube is flushed several times with the test gas and then pumped again. Along the shock tube, three ion probes denoted as No.1, No.2 and No.3 are equipped, and the shock velocity is estimated from the probe signals recorded on a digital oscilloscope. The shock velocities in these runs are close to $6.31 \mathrm{~km} / \mathrm{s}$ and vary about $1.7 \%$ on average. Two $10 \mathrm{~mm}$ diameter quartz windows are equipped on both sides of the shock tube to carry out optical accessible measurements.

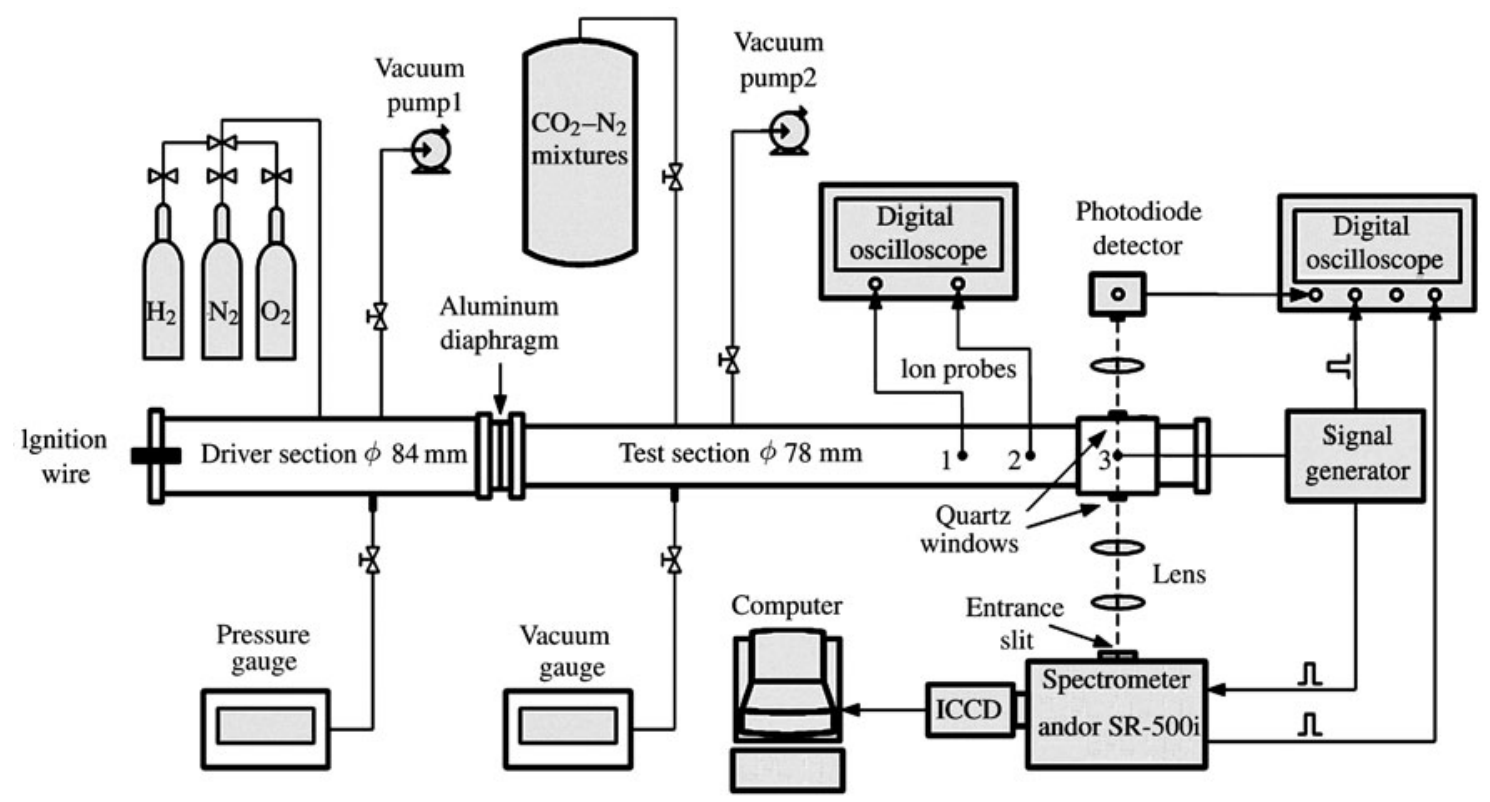

Fig. 1 Schematic diagram of the shock tube and the emission spectroscopy measurement setup

The optical system consists of a spectrometer (Model: SR-500i, $500 \mathrm{~mm}$ focal length, Andor technology) and an ICCD camera (Model: DH740, $2048 \times 512$ pixels, Andor technology). The spectrometer used is equipped with a
1200 grooves/mm grating and an entrance slit with its width equals to $100 \mu \mathrm{m}$ to get an acceptable signal-to-noise ratio and a sufficient spectral resolution. The full-width at halfmaximum (FWHM) of the apparatus function is measured 
experimentally to be Lorentzian profile of value $0.154 \mathrm{~nm}$ by using of a narrow line width semiconductor diode laser (center wavelength: $777.19 \mathrm{~nm}, 10 \mathrm{MHz}$ line width). We use the apparatus function as the spectral function, this is because most broadening mechanisms, such as Doppler, natural, resonance and others, are negligible in comparison with the instrumental broadening in our experimental conditions. Wavelength calibration for each grating is carried out by comparison with the emission lines of an $\mathrm{Hg}$ lamp. An NIST traceable quartz-tungsten-halogen standard light source (Model: 63945, Oriel Corporation) is used for irradiance calibration. Due to the large size of the standard light, irradiance calibration is carried out outside the shock tube; however, the same glass windows as used in the experiments are placed between the lamp and the spectrometer to obtain calibration data including the effect of window absorption. Thus, we use a relative irradiance scale in this study. When the shock wave arrives at the location of No.3 probe which is installed on the same section as spectrum capture field, the optical system is triggered by the output of the ion probe through a signal generator (Model: DG535, Stanford Research System). The ion probe has a very quick response ( $\sim 100 \mathrm{~ns})$ to the arrival of the shock waves. The internal time delay of electric circuit in the ICCD system is $40 \mathrm{~ns}$. The spectra capture field is strictly controlled with $1 \mathrm{~mm}$ in diameter and $1 \mathrm{~mm}$ in depth by the lens system installed in front of the spectrometer. For each experimental run, the camera exposure is set to $50 \mathrm{~ns}$ in order to acquire adequate temporal resolution. Although only one single spectrum can be recorded during a shot, the delay of the gate pulse can be tuned to record different moments in which the spectrum is to be measured in the equilibrium, or in the non-equilibrium region. A photodiode detector is used to monitor the radiation profile of the high temperature gas behind the strong shock wave. The signal output from ion probes and photodiode are recorded by a high dynamic response digital oscilloscope (Model: DPO4034, Tektronix Inc.).

\section{Measurements and uncertainty analysis}

3.1 Temperature determination and rotational temperature deviation evaluation

Generally, the routine method of rotational and vibrational temperature measurement by comparison between the observed and theoretical synthetic spectra has been widely adopted as many literatures refer to. However, in the previous studies, the spectrum resolution is always limited by the acquisition range of the spectroscopy apparatus, that high resolution to separate individual rotational lines must be sacrificed for a wide range of acquisition wavelength. As a result this method depends strongly on the accuracy of calculated spectra and the accurate calibration of the instrument function. In our study, we analyze in detail the spectral structure of $\mathrm{CN}$ violet system $\left(B^{2} \Sigma^{+} \rightarrow X^{2} \Sigma^{+}, \Delta v=0\right.$ sequence) and propose a rotational and vibrational temperature measurement method based on fitting high-resolution spectrum.
In the study, we focus on the ranges $(387.6-388.6 \mathrm{~nm})$ of the band of the $\mathrm{CN}$ violet system $\left(B^{2} \Sigma^{+} \rightarrow X^{2} \Sigma^{+}, \Delta v=0\right.$ sequence), this wavelength range corresponds to the $(0,0)$ transition of the $\mathrm{CN}$ violet system $\left(B^{2} \Sigma^{+} \rightarrow X^{2} \Sigma^{+}, \Delta v=0\right.$ sequence), as shown in Fig. 2a. The rectangular panel shows that the range of wavelength is completely controlled by emission lines of the $P$ branch (rotational number $J$ range from 0 to 57), in other words, this distribution pattern of a sequence is controlled only by rotational temperature. We call this fraction of the wavelength range the "bandhead $G_{0}$ ", the effect of FWHM of other rotational lines tends to be neglected, as shown in Fig. 2b. So the rotational temperature is determined by comparing the shape of bandhead $G_{0}$ between the experimental and theoretical synthetic spectra. Then, the vibrational temperature is estimated by minimizing the difference between the observed and theoretical spectra.
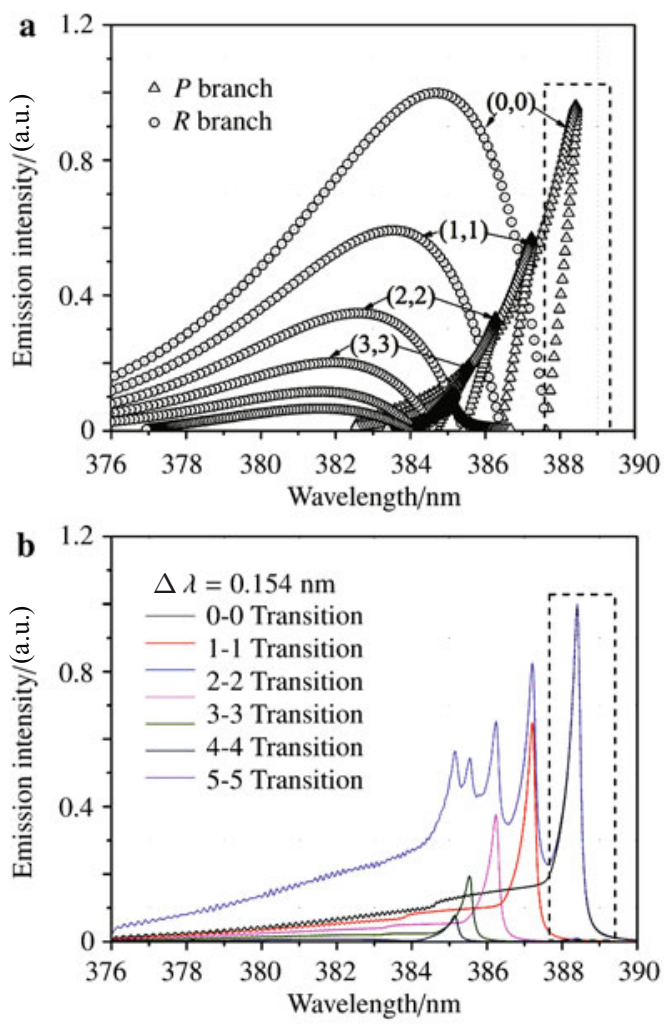

Fig. 2 Structure of a rotational lines and $\mathbf{b}$ theoretical synthetic spectra in the $\mathrm{CN}$ violet band

In the error evaluation of the rotational temperature measurement, we investigate the fitting errors from the theoretical uncertainty that stems from the spectroscopic constants. As discussed above, the bandhead $G_{0}$ is controlled only by $T_{\text {rot }}$, so the most influential spectroscopic constant is the rotational constant $B_{\mathrm{e}}$, and the spectroscopic constants of the vibrational term such as $\omega_{\mathrm{e}} x_{\mathrm{e}}$, while $\omega_{\mathrm{e}} y_{\mathrm{e}}$, and $\omega_{\mathrm{e}} z_{\mathrm{e}}$ are not relevant for $T_{\text {rot }}$ using the bandhead $G_{0}$ fitting method. We discuss in detail the reason as follows. 
The emission intensity is described as

$I_{v^{\prime \prime}, J^{\prime \prime}}^{v^{\prime}, J^{\prime}}=h v_{v^{\prime \prime}, J^{\prime \prime}}^{v^{\prime}, J^{\prime}} N_{v^{\prime}, J^{\prime}} A_{v^{\prime \prime}, J^{\prime \prime}}^{v^{\prime}, J^{\prime}}$

Assuming the Boltzmann distribution at the ground state, so the population at $\left(v^{\prime}, J^{\prime}\right)$ state is described as

$N_{v^{\prime}, j^{\prime}} \propto\left(2 J^{\prime}+1\right) g_{J^{\prime}} \exp \left(-\frac{F\left(v^{\prime}, J^{\prime}\right)}{k T_{\text {rot }}^{\prime}}-\frac{G\left(v^{\prime}\right)}{k T_{\text {vib }}^{\prime}}\right)$.

Fixing the value of $v^{\prime}$ and $v^{\prime \prime}$, taking the logarithm of both sides, substituting Eq. (2) to Eq. (1) and taking the logarithm of both sides, we obtain

$\lg \left[\frac{I_{J^{\prime \prime}}^{J^{\prime}}}{v_{J^{\prime \prime}}^{J^{\prime}} A_{J^{\prime \prime}}^{J^{\prime}}\left(2 J^{\prime}+1\right) g_{J^{\prime}}}\right]=-\frac{F\left(v^{\prime}, J^{\prime}\right)}{k T_{\text {rot }}^{\prime}}+c_{1}$,

where $A_{J^{\prime \prime}}^{J^{\prime}}$ can be calculated explicitly [14], which will be discussed in detail in the appendix, $c_{1}$ is a constant.

$$
\begin{aligned}
& F\left(v^{\prime}, J^{\prime}\right)=B_{v^{\prime}} J^{\prime}\left(J^{\prime}+1\right)-D_{v^{\prime}} J^{2}\left(J^{\prime}+1\right)^{2}, \\
& B_{v^{\prime}}=B_{\mathrm{e}}-\alpha_{\mathrm{e}}\left(v^{\prime}+\frac{1}{2}\right), \\
& D_{v^{\prime}}=D_{\mathrm{e}}+\beta_{\mathrm{e}}\left(v^{\prime}+\frac{1}{2}\right), \\
& v_{v^{\prime \prime}, J^{\prime \prime}}^{v^{\prime}, J^{\prime}}=v_{v^{\prime}, v^{\prime \prime}}+F\left(J^{\prime}\right)-F\left(J^{\prime \prime}\right) .
\end{aligned}
$$

The rotational quantum numbers corresponding to the wavelengths of the $\mathrm{CN}$ bandhead $G_{0}$ range from 0 to 57 for the $(0,0)$ transition. In this case, both the first term of Eqs. (4) and (5) are at least 90 times the second term, and thus the second terms of them are neglected and Eq. (4) can be written as

$F\left(J^{\prime}\right)=B_{\mathrm{e}} J^{\prime}\left(J^{\prime}+1\right)$.
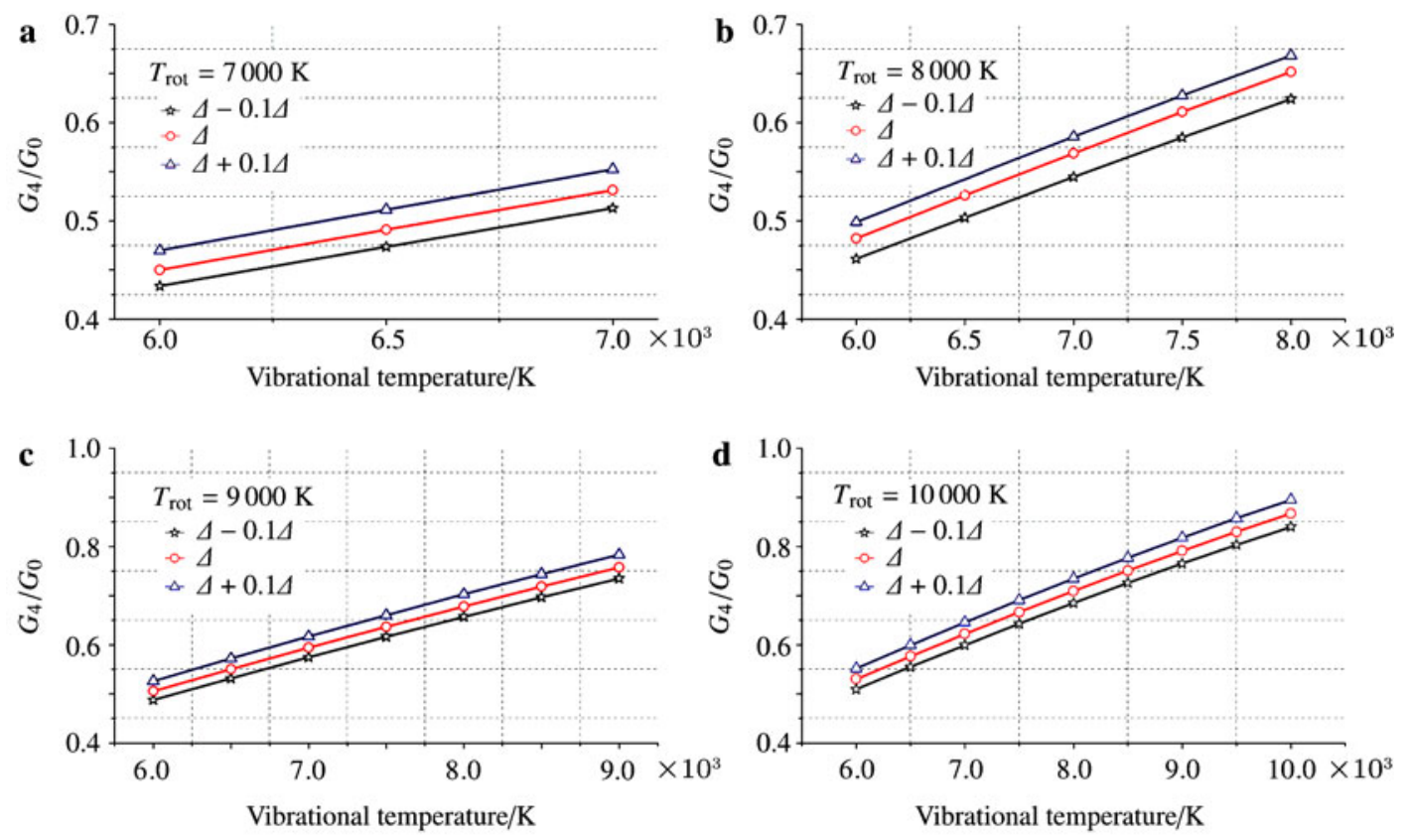

Fig. 3 Peak ratio as a function of $T_{\text {vib }}$ for estimating $T_{\text {vib }}$ by spectra synthesis (the slit function is Lorentzian profile as measured)
The first term of the wavenumber of each rotational line is much larger than the second term, and $v_{v^{\prime}, v^{\prime \prime}}$ is a constant for a given electronic transition, so the uncertainty due to $B_{\mathrm{e}}$ in $v_{v^{\prime \prime}, J^{\prime \prime}}^{v^{\prime}, J^{\prime}}$ is negligible, and Eq. (3) can be described as

$\lg \left(\frac{I_{J^{\prime \prime}}^{J^{\prime}}}{c_{3}}\right)=-\frac{c_{2} B_{\mathrm{e}}}{T_{\text {rot }}^{\prime}}+c_{1}$

where $c_{2}$ and $c_{3}$ are two constants.

From above discussion, we know $B_{\mathrm{e}}$ is the dominant spectroscopic constant affecting the emission intensity. Consequently, as shown in Eq. (9), the systematic error in $T_{\text {rot }}$ is of the same order of magnitude as that of $B_{\mathrm{e}}$. We know the difference in $B_{\mathrm{e}}$ is less than $1 \%$ by relevant literature [15-17], so the systematic error in $T_{\text {rot }}$ is also less than $1 \%$. Therefore, the result shows that the uncertainties in spectroscopic constants have ignorable effect on the observed rotational temperature in our method.

3.2 Effect of spectral resolution on vibrational temperature measurement

In this section, we use the ratio between bandhead $G_{4}$ and bandhead $G_{0}$ to estimate the $T_{\text {vib }}$ error due to the measurement uncertainty of spectral resolution $\Delta$. Figure 3 shows the peak ratio of $G_{4}$ to $G_{0}$ as a function of $T_{\text {vib }}$ for three values of FWHM as the rotational temperature changes from $8000 \mathrm{~K}$ to $12000 \mathrm{~K}$. Assume the uncertainty on the spectral broadening is $\pm 10 \%$, that is to say our spectral resolution $\triangle$ changes from $0.139 \mathrm{~nm}$ to $0.170 \mathrm{~nm}$. Figure $3 \mathrm{f}$ shows that

Springer 

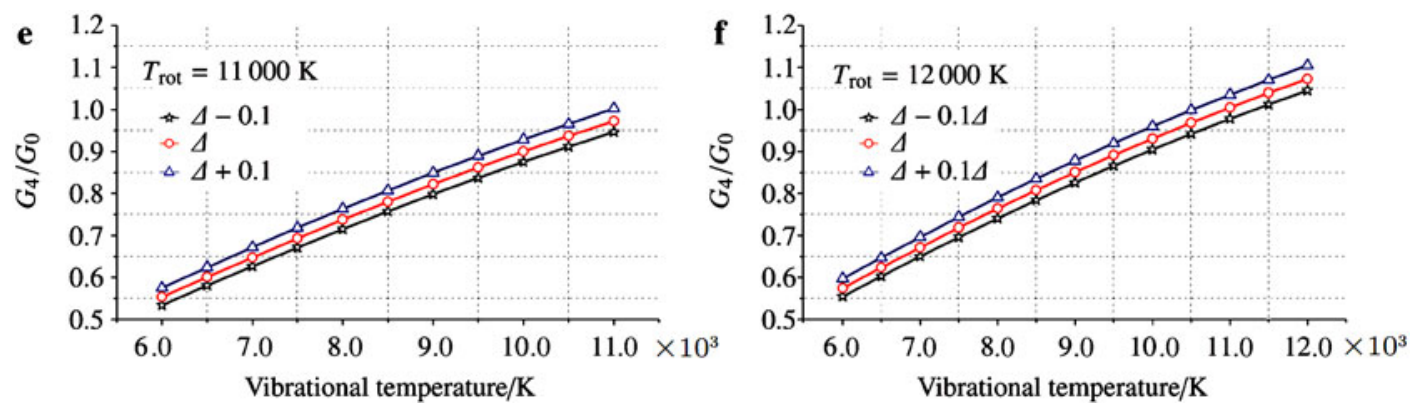

Fig. 3 Peak ratio as a function of $T_{\text {vib }}$ for estimating $T_{\text {vib }}$ by spectra synthesis (the slit function is Lorentzian profile as measured) (continued)

$T_{\text {vib }}$ is slightly affected by the FWHM when the rotational temperature is at $12000 \mathrm{~K}$, the error range is from $-110 \mathrm{~K}$ to $210 \mathrm{~K}$. Considering the reduction of the slope of the curve due to the decreasing rotational temperature, as shown in Figs. 3, the $T_{\text {vib }}$ error may increase but the maximum does not exceed $8 \%$ on average in the experimental condition.

\section{Results and discussions}

\subsection{Rotational temperature determination}

With the ICCD gating time of $50 \mathrm{~ns}$, spectra have been obtained each at $200 \mathrm{~ns}$ interval when the medium is considered out of equilibrium and each at $2 \mu$ s when equilibrium is assumed. First, spectral analysis is carried out for the ranges (387.6-388.6 $\mathrm{nm}$ ) of the band of the $\mathrm{CN}$ violet system $\left(B^{2} \Sigma^{+} \rightarrow X^{2} \Sigma^{+}, \Delta v=0\right.$ sequence). The rotational temperature is then determined by the bandhead $G_{0}$ fitting method. Figure 4 shows a representative comparison between an observed $\mathrm{CN}$ spectrum and three theoretical synthetic spectra for different rotational temperatures, regardless of the value of $T_{\text {vib }}$. The black and red lines represent the theoretical synthetic spectra with the rotational temperatures of $8700 \mathrm{~K}$ and $7600 \mathrm{~K}$, respectively. The dashed line shows the theoretical synthetic spectra when $T_{\text {rot }}$ is fixed at the optimum value. In Fig. 4, the bandhead $G_{0}$ of the observed and theoretical

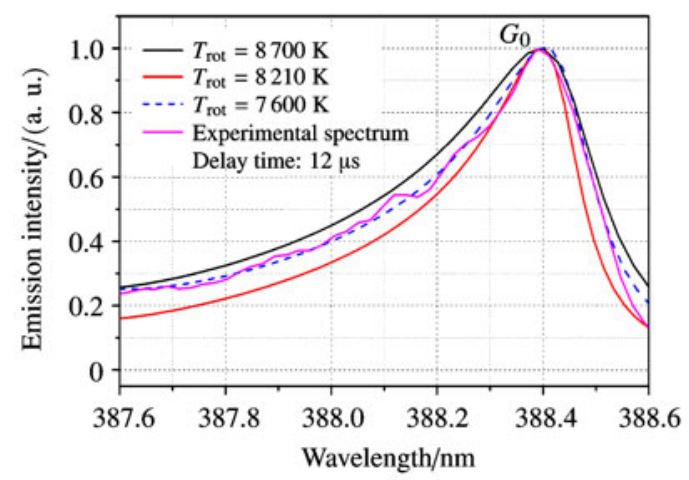

Fig. 4 Comparison of an observed $\mathrm{CN}$ bandhead $G_{0}$ with three theoretical synthetic spectra for different rotational temperatures synthetic spectra are in good agreement. Also, significant differences can be seen if we change $T_{\text {rot }}$ in a small range.

4.2 Spectra analysis in the non-equilibrium and equilibrium region

Figure 5 presents some comparisons of experimental spectra with calculated spectra when non-equilibrium and equilibrium are assumed to be achieved, respectively. In order to describe the measurement accuracy, we use the quantity $E_{r}$ to assess the degree of difference between the experimental and calculated spectra [18], which is defined as follows

$E_{r}=\frac{\left(I_{\mathrm{cal}}(\lambda)-I_{\exp }(\lambda)\right)^{2}}{\left(I_{\exp }(\lambda)\right)^{2}}$.

Figure 5a shows some discrepancy between the theoretical synthetic and experimental spectra in the wavelength range of 376-385 $\mathrm{nm}$ (the tail region of $\mathrm{CN}$ violet band), which demonstrates that the vibrational state of $\mathrm{CN}$ radicals is unlikely to have a Boltzmann distribution for high $J$ rotational lines (it can also be found in the error estimation figure). A disturbed distribution of vibrational states affects the spectral shape, especially in the tail region of the $\mathrm{CN}$ spectra. This is because the characteristic time scale of the vibrational relaxation of $\mathrm{CN}(\sim 1 \mu \mathrm{s})[19]$ is much longer than that of the radiation due to the electronic transition of the $\mathrm{CN}$ violet band system $(\sim 60 \mathrm{~ns})[20]$. But for wavelength in the range of 385-390 nm, the synthetic spectra agree well with experimental spectra. The tail region of spectra $(376-385 \mathrm{~nm})$ is unlikely to have large influence on vibrational temperature determination due to much lower energy quotient in high $J$ rotational line states compared with energy quotient in low $J$ rotational line states, which can also be confirmed through integration of Eq. (10) over the whole wavelength range for deviation evaluation. Therefore, the vibrational temperature determination can still keep satisfactory precision even in thermochemical non-equilibrium state. In the quasi equilibrium and equilibrium region, as shown in Figs. $5 \mathrm{~b}$ and $5 \mathrm{c}$, the results indicate that the theoretical synthetic and experimentally measured spectra match up very well and reasonable temperature fitting has been achieved. 

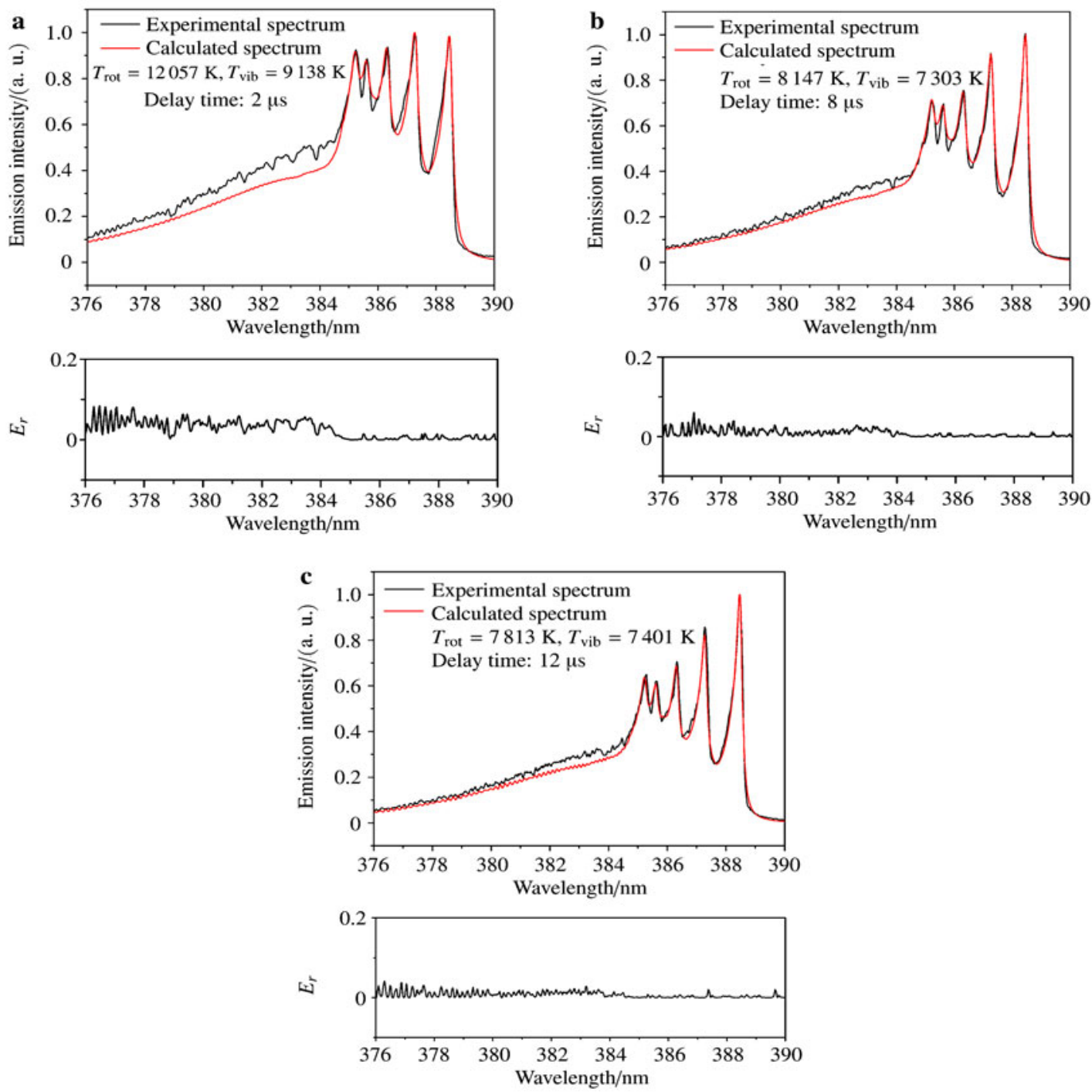

Fig. 5 Experimental and theoretical synthetic spectra of $\mathrm{CN}$ violet system $(\Delta v=0)$ at different delay times. (Experiment: $P_{1}=200 \mathrm{~Pa}$, $V_{\text {shock }}=6.31 \pm 0.11 \mathrm{~km} / \mathrm{s}$. Composition: $70 \% \mathrm{CO}_{2}$ and $30 \% \mathrm{~N}_{2}$.)

4.3 Evolution of vibrational and rotational temperatures behind the shock wave

Comparisons between these experimental spectra and the calculated ones allow the temporal profile of the temperatures behind the shock wave to be drawn. Figure 6 presents the evolution of vibrational and rotational temperatures behind the shock wave, where $t_{\mathrm{p}}$ is the induction time behind a shock wave and $t_{\mathrm{e}}$ is defined as the relaxation time of non-equilibrium region. For the current experimental conditions, considering the response of ion probe and time delay of ICCD system, the $t_{\mathrm{p}}$ and $t_{\mathrm{e}}$ have values of $(2.0 \pm 0.2) \mu \mathrm{s}$ and $14 \pm 1.0 \mu \mathrm{s}$, respectively. From the previous analysis and the temperature distribution behind the shock wave, the shock heated gas should reach equilibrium after $16 \mu \mathrm{s}$. The uncertainty is equal to $800 \mathrm{~K}$ in the non-equilibrium in the first $4 \mu \mathrm{s}$, and to $400 \mathrm{~K}$ in the equilibrium after next $12 \mu \mathrm{s}$ on both temperatures.

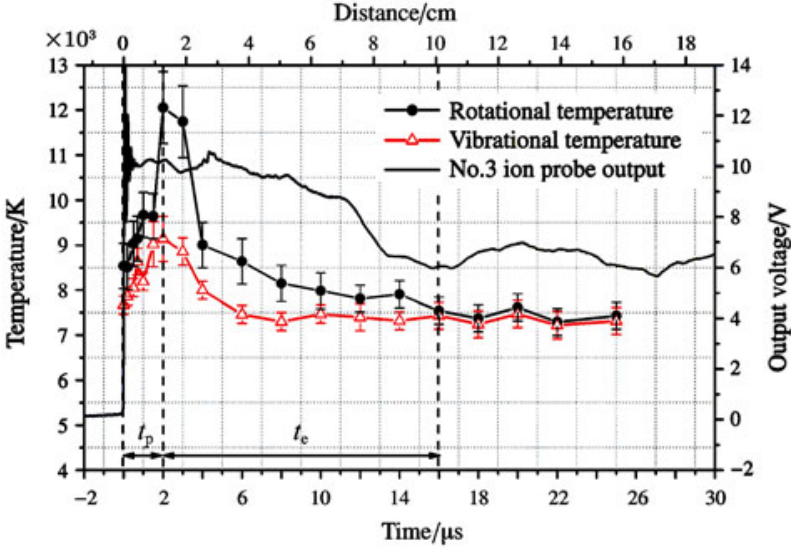

Fig. 6 Evolution of vibrational and rotational temperatures behind the shock wave. (Experiment: $P_{1}=200 \mathrm{~Pa}, V_{\text {shock }}=6.31 \pm$ $0.11 \mathrm{~km} / \mathrm{s}$. Composition: $70 \% \mathrm{CO}_{2}$ and $30 \% \mathrm{~N}_{2}$.) 


\section{Conclusions}

Non-equilibrium radiation measurements behind strong shock wave for simulated Martian atmosphere are presented in this paper. The shock wave is established in a hydrogen oxygen combustion driven shock tube. Time-resolved spectra of the $\Delta v=0$ sequence of the $B^{2} \Sigma^{+} \rightarrow X^{2} \Sigma^{+}$electronic transition of $\mathrm{CN}$ have been observed through optical emission spectroscopy (OES). We analyze in detail the spectral structure of $\mathrm{CN}$ violet system $\left(B^{2} \Sigma^{+} \rightarrow X^{2} \Sigma^{+}, \Delta v=0\right.$ sequence ) and propose a rotational and vibrational temperatures measurement method based on fitting high-resolution spectrum. The theoretical spectrum calculation error due to uncertainties in the spectroscopic constants and the measurement of spectral revolution FWHM has been systematically analyzed. Radiation structure of the shock layer, including induction, relaxation and equilibrium process, and corresponding rotational and vibrational temperature are obtained through analysis of time gating optical emission spectra with sub-microsecond temporal resolution. The present extensive results will strongly benefit the reaction rate estimation and CFD code validation in high enthalpy Mars reentry chemistry.

\section{References}

1 Bose, D., Wright, M. J., Bogdanoff, D. W., et al.: Modeling and experimental assessment of $\mathrm{CN}$ radiation behind a strong shock wave. Journal of Thermophysics and Heat Transfer 20, 220-230 (2006)

2 Braun, R. D., Manning, R. M.: Mars exploration entry, descent, and landing challenges. Journal of Spacecraft and Rockets 44, 310-323 (2007)

3 Park, C., Howe, J. T., Jaffe, R. L., et al.: Review of chemical kinetic problems of future nasa mission II: mars entries. Journal of Thermophysics and Heat Transfer 8, 9-23 (1994)

4 Playez, M., Fletcher, D. G.: Spectroscopic analysis of titan atmospheric plasmas. Journal of Thermophysics and Heat Transfer 22, 150-156 (2008)

5 Bultel, A., Chéron, B. G., Bourdon, A., et al.: Collisionalradiative model in air for earth re-entry problems. Physics of Plasmas 13, 043502 (2006)

6 Rond, C., Boubert, P.: Chemical kinetic and radiative simulations for titan atmospheric entry. Journal of Thermophysics and Heat Transfer 23, 72-82 (2009)
7 Gökcen, T.: $\mathrm{N}_{2}-\mathrm{CH}_{4}-\mathrm{Ar}$ chemical kinetic model for simulations of atmospheric entry to titan. Journal of Thermophysics and Heat Transfer 21, 9-18 (2007)

8 Labracherie, L., Billiotte, M., Houas, L.: Shock-tube analysis of argon influence in titan radiative environment. Journal of Thermophysics and Heat Transfer 10, 162-168 (1996)

9 Grinstead, J. H., Wright, M. J., Bogdanoff, D. W., et al.: Shock radiation measurements for Mars aerocapture radiative heating analysis. Journal of Thermophysics and Heat Transfer 23, 249255 (2009)

10 Bose, D., Grinstead, J. H, Bogdanoff, D. W., et al.: Shock layer radiation measurements and analysis for mars entry. In: Proceedings of the 3rd International Workshop on Radiation of High Temperature Gases in Atmospheric Entry Heraklion, Greece (2009)

11 Boubert, P., Rond, C.: Nonequilibrium radiation in shocked martian mixtures. Journal of Thermophysics and Heat Transfer 24, 40-49 (2010)

12 Rond, C., Boubert, P., Félio, J. M., et al.: Radiation measurements in a shock tube for titan mixtures. Journal of Thermophysics and Heat Transfer 21, 638-646 (2007)

13 Aaron, M. B., Richard, G. M., Timothy, J. M., et al.: Nonequilibrium radiation intensity measuerments in simulated titan atmospheres. Journal of Thermophysics and Heat Transfer 24, 291-300 (2010)

14 Hertzberg, G.: Molecular Spectra and Molecular Structure IV: Constants of Diatomic Molecules. van Nostrand Reinhold, New York (1979)

15 Herzberg, G.: Molecular Spectra and Molecular Structure I: Spectra of Diatomic Molecules. Malabar, Florida (1989)

16 Ram, R. S, Davis, S. P, Wallace, L., et al.: Fourier transform emission spectroscopy of the $B^{2} \Sigma^{+}-X^{2} \Sigma^{+}$system of CN. Journal of Molecular Spectroscopy 237, 225-231 (2006)

17 Praced, C. V., Bernath, P. F.: Fourier transform jet-emission spectroscopy of the $A^{2} \Pi^{+}-X^{2} \Sigma^{+}$transition of CN. Journal of Molecular Spectroscopy 156, 327-340 (1992)

18 Kurosawa, K., Sugita, S., Fujita, K., et al.: Rotationaltemperature measurements of chemically reacting $\mathrm{CN}$ using band-tail spectra. Journal of Thermophysics and Heat Transfer 23, 463-472 (2009)

19 Slack, M. W.: Kinetics and thermodynamics of the $\mathrm{CN}$ molecule. iii. shock tube measurement of $\mathrm{CN}$ dissociation rates. Journal of Chemical Physics 64, 228-236 (1976)

20 Knowles, P. J, Werner, H. J., Cartwright D. C., et al.: The $A^{2} \Pi^{+}-X^{2} \Sigma^{+}$red and $\mathrm{B}^{2} \Sigma^{+}-X^{2} \Sigma^{+}$violet systems of the $\mathrm{CN}$ radical: Accurate multireference configuration interaction calculations of the radiative transition probabilities. Journal of Chemical Physics 89, 7334-7343 (1988) 